\title{
Building up resilience in an uncertain world: mental health challenges in the aftermath of the first modern pandemic
}

\author{
Marta Rapado-Castro ${ }^{1,2} \cdot$ Celso Arango $^{1}$
}

Published online: 4 August 2021

๑) Springer-Verlag GmbH Germany, part of Springer Nature 2021

Now more than ever, preventative and interventional strategies need to be put in place to overcome the collateral damage caused by the pandemic. Mental health is one of the casualties. A long time has passed since the Wuhan outbreak, and the world is or will be soon experiencing the aftermath of the first modern pandemic. Now is, therefore, the time to take notice of the effects of sustained stress on mental and physical health over time and to take action to address them.

The uncertainty of the COVID-19 pandemic has been a challenge to mental health globally, transforming the way people make sense of the world and how they relate to one another. The pandemic has presented us with both physical and mental challenges, including the continued stress of lockdowns, restrictions, social distancing, job losses, income insecurity, health and social inequality, suffering, and isolation. Provision of services has changed [1], and the consequences of the pandemic for mental health have become particularly evident in vulnerable groups such as people with pre-existing mental health conditions and individuals at risk for mental disorders, children and adolescents in particular. The incidence of stress-related mental disorders has increased throughout the pandemic, with suicidal crises, anxiety disorders, eating disorders, and major depressive episodes been the most compelling in this population [2]. A recent survey to estimate the impact of the COVID-19 pandemic on child and adolescent psychiatry services in Europe [2] found a dramatic increase in referrals for assessment and hospitalizations 1 year into the pandemic, with perception of

Marta Rapado-Castro

mrapado@iisgm.com

1 Department of Child and Adolescent Psychiatry, Institute of Psychiatry and Mental Health, Hospital General Universitario Gregorio Marañón, School of Medicine, Universidad Complutense, IiSGM, CIBERSAM, Madrid, Spain

2 Department of Psychiatry, Melbourne Neuropsychiatry Centre, The University of Melbourne and Melbourne Health, 161 Barry Street, Carlton South, VIC 3053, Australia the impact of the pandemic on mental health and psychopathology in this population escalating from moderate in 2020 to extreme in 2021.

In this context, lessons must be learnt about mechanisms of cope, resilience, and adaptation. As Chmitorz et al. [3] noted in this issue of European Archives of Psychiatry and Clinical Neuroscience, there is an urgent need to investigate protective mechanisms that support the maintenance of mental health during and after adversity. These authors focus on cognitive, physiological, and neural pathways (i.e., resilience mechanisms) that may provide protection against stress-related impairments in a large sample of 1191 individuals. They present baseline descriptive data from the ongoing Longitudinal Resilience Assessment (LORA) study, a population-based study aiming to characterize resilience as a continued process of biological, neural, and cognitive adaptation to stressors and daily hassles, directly assessing their influence on the human organism over a period of at least 3 years. It is very timely that these authors are using a comprehensive, granular approach to the investigation of the general (lifestyle, cortisol reactivity, microbiome, genetic, epigenetic and cognitive) mechanisms involved in resilience in adults from the community, performing an in-depth exploration of their mental health status, ability to recover from stress, major life events, daily hassles, and perceived stress mechanisms, with the aim of clarifying the cognitive and biological mechanisms that may determine whether or not clinical psychopathology develops.

It is well known that long-term exposure to stress is involved in and increases the risk of mental disorders. However, the factors that influence stress levels and regulatory mechanisms of the brain are not well understood. Some of the previously suggested plausible biological and cognitive contributing factors to the stress and resilience response, such as cortisol reactivity and perceived anxiety, have been investigated before. For instance, recent findings point to a blunted cortisol stress response and greater perceived anxiety in a dose-response relationship in children 
and adolescents exposed to early adversity [4]. In particular, exposure to maltreatment may impact stress response by biological systems involved in emotional processing in major disorders such as psychosis. In this issue, Quidé et al. [5] report on the relationship between cortisol reactivity and social brain function in three groups of individuals: subjects with schizophrenia or bipolar disorder, and controls. They found that exposure to childhood trauma moderates the associations between cortisol reactivity and neural correlates of emotion processing in psychotic disorders. Moreover, they found distinctive moderating effects of childhood trauma exposure on the relationship between neuroendocrine response and brain function associated with emotion processing in schizophrenia, bipolar disorder, and controls, suggesting differential impact of stress on mechanisms of emotion regulation among these three groups. Their findings may have implications for the development of novel diagnosis-specific interventions directed at mitigating the effects of stress in psychosis.

Individuals with psychosis have consistently reported higher perceived stress levels than controls [6]. The impact of potential risk factors such as cognitive functioning and coping styles on perceived stress has previously been investigated in this context, with specific contributions of higher cognitive function and greater use of an emotion-focused coping style to elevated stress in psychosis. On the contrary, lower premorbid cognitive functioning together with higher levels of emotion-focused coping were associated with increased perceived stress in controls [6]. Along these lines, the study by Evermann et al. [7] in this issue shows that distress severity moderates the relationship between the presence of psychotic-like experiences and brain volumes in non-help-seeking individuals from the community. Furthermore, this study highlights the role of perceived stress in the interaction between psychotic-like experiences and prefrontal brain abnormalities that may lead to the development of psychotic disorders. Brain volume reductions, particularly in prefrontal regions, have consistently been found in individuals with psychosis. Moreover, previous studies suggest that the morphology of prefrontal and temporal structures may be particularly vulnerable to the stress of childhood trauma with potential predictive value for transition to psychosis in individuals at ultra-high risk (UHR) for psychosis [8]. These studies support the need to target various types of stressors and/or childhood trauma in both the assessment and treatment of individuals at risk for major disorders. Treatment approaches that take history of stress into account may be useful to mitigate both its physical and psychosocial effects in these subjects.

In this vein, the study by Wullschleger et al. [9] in this issue examines the impact of standardized post-coercion review sessions as an easy-to-implement preventative interventions directed at mitigating the experience of distress and post-traumatic symptoms in individuals with psychosis who underwent coercive measures (e.g., mechanical restraint or isolation) in an inpatient setting, using a randomized controlled trial design. Coercion in psychiatric care is a potentially traumatic experience leading to the development of pervasive and invalidating post-traumatic symptoms. A total of $70 \%$ of participants in the Wullschleger et al. [9] study presented post-traumatic reactions to coercion with $20 \%$ having a high probability of post-traumatic stress disorder (PTSD), highlighting the importance of careful management of these experiences and related PTSD. These authors demonstrated that post-coercion review sessions can be an effective way to overcome distress and prevent the development of PTSD symptoms in individuals with psychosis in comparison with the control group. Their results further emphasize the need to provide assessment and damage control for potentially negative effects of inpatient coercive measures that may have long-term consequences on the course of psychosis.

In sum, these are particularly challenging times when small targeted stress management interventions may have a significant impact on the course of mental disorders. We know about the effects of stress on the brain and physical symptoms and how they relate to the development of psychiatric conditions. At a time when daily chronic stressors and sustained social and economic restrictions implemented to prevent the spread of the COVID-19 may have dramatic cumulative effects on mental health, extra support is crucial to mitigate the negative consequences of sustained stressors in particularly vulnerable populations [10].

Overall, pandemics may constitute a time of particularly sensitive changes and adaptation to stress with relevance for the course and development of mental health conditions, but also present a significant opportunity for a paradigm shift in mental health research and service delivery. Focusing on development of newer preventative interventions, stress management assessment, and potentiation of resilience mechanisms could help us take advantage of this opportunity and mitigate the mental health effects of the pandemic. Since stress is involved in the development and course of mental disorders, improved understanding of the multisystem factors that may contribute to elevated stress response in individuals will undoubtedly further important clinical and societal goals, particularly relevant in the aftermath of the COVID-19 pandemic.

Acknowledgements MR-C is a Ramon y Cajal Research Fellow (RYC2017-23144), Spanish Ministry of Science, Innovation and Universities and was supported by a NARSAD independent investigator grant (No. 24628) from the Brain \& Behavior Research Foundation. MR-C and CA were partially supported by the Spanish Ministry of Science and Innovation, Instituto de Salud Carlos III, (PI18/00753, PI21/00701), co-financed by ERDF Funds from the European Commission, "A 
way of making Europe", CIBERSAM, Madrid Regional Government (B2017/BMD-3740 AGES-CM-2), European Union Structural Funds, European Union Seventh Framework Program, Fundación Familia Alonso, and Fundación Alicia Koplowitz.

\section{Declarations}

Conflict of interest CA has been a consultant to or has received honoraria or grants from Acadia, Angelini, Boehringer, Gedeon Richter, Janssen-Cilag, Lundbeck, Minerva, Otsuka, Roche, Sage, Servier, Shire, Schering-Plough, Sumitomo Dainippon Pharma, Sunovion, and Takeda, for work unrelated to the submitted work.

\section{References}

1. Moreno C, Wykes T, Galderisi S, Nordentoft M, Crossley N, Jones N, Cannon M, Correll CU, Byrne L, Carr S, Chen EYH, Gorwood P, Johnson S, Kärkkäinen H, Krystal JH, Lee J, Lieberman J, López-Jaramillo C, Männikkö M, Phillips MR, Uchida H, Vieta E, Vita A, Arango C (2020) How mental health care should change as a consequence of the COVID-19 pandemic. Lancet Psychiatry 7(9):813-824

2. Revet A, Hebebrand J, Anagnostopoulos D, Kehoe LA, GradlDietsch G; COVID-19 Child and Adolescent Psychiatry Consortium, Klauser P (2021) Perceived impact of the COVID-19 pandemic on child and adolescent psychiatric services after 1 year (February/March 2021): ESCAP CovCAP survey. Eur Child Adolesc Psychiatry. 29:1-8. https://doi.org/10.1007/ s00787-021-01851-1

3. Chmitorz A, Neumann RJ, Kollmann B (2020) Longitudinal determination of resilience in humans to identify mechanisms of resilience to modern-life stressors: the longitudinal resilience assessment (LORA) study. Eur Arch Psychiatry Clin Neurosci. https://doi.org/10.1007/s00406-020-01159-2

4. Marques-Feixa L, Palma-Gudiel H, Romero S, Moya-Higueras J, Rapado-Castro M, Castro-Quintas Á, Zorrilla I, José Muñoz M,
Ramírez M, Mayoral M, Mas A, José Lobato M, Blasco-Fontecilla H, Fañanás L; EPI-Young Stress GROUP (2021) Childhood maltreatment disrupts HPA-axis activity under basal and stress conditions in a dose-response relationship in children and adolescents. Psychol Med 16:1-14. https://doi.org/10.1017/S00332917210024 9X

5. Quidé Y, Girshkin L (2020) The relationship between cortisol reactivity and emotional brain function is differently moderated by childhood trauma, in bipolar disorder, schizophrenia and healthy individuals. Eur Arch Psychiatry Clin Neurosci. https://doi.org/ 10.1007/s00406-020-01190-3

6. Allott KA, Rapado-Castro M, Proffitt TM, Bendall S, Garner B, Butselaar F, Markulev C, Phassouliotis C, McGorry PD, Wood SJ, Cotton SM, Phillips LJ (2015) The impact of neuropsychological functioning and coping style on perceived stress in individuals with first-episode psychosis and healthy controls. Psychiatry Res 226(1):128-135

7. Evermann U, Schmitt S (2021) Distress severity in perceptual anomalies moderates the relationship between prefrontal brain structure and psychosis proneness in nonclinical individuals. Eur Arch Psychiatry Clin Neurosci. https://doi.org/10.1007/ s00406-020-01229-5

8. Rapado-Castro M, Whittle S, Pantelis C, Thompson A, Nelson B, Ganella EP, Lin A, Reniers R, McGorry PD, Yung AR, Wood SJ, Bartholomeusz CF (2020) Does cortical brain morphology act as a mediator between childhood trauma and transition to psychosis in young individuals at ultra-high risk? Schizophr Res 224:116-125

9. Wullschleger A, Vandamme A, Mielau J, Renner L, Bermpohl F, Heinz A, Montag C, Mahler L (2020) Effect of standardized post-coercion review session on symptoms of PTSD: results from a randomized controlled trial. Eur Arch Psychiatry Clin Neurosci. https://doi.org/10.1007/s00406-020-01215-x

10. Stefana A, Youngstrom EA, Hopwood CJ, Dakanalis A (2020) The COVID-19 pandemic brings a second wave of social isolation and disrupted services. Eur Arch Psychiatry Clin Neurosci 270(6):785-786. https://doi.org/10.1007/s00406-020-01137-8 